\title{
Usefulness of Immuno-Magnetic Beads Conjugated with Anti-EpCAM Antibody for Detecting Endometrial Cancer Cells*
}

\author{
Yoshikatsu Koga $^{1}$, Satoshi Katayose ${ }^{2}$, Nobuko Onoda ${ }^{2}$, Takahiro Kasamatsu ${ }^{3}$, Tomoyasu Kato ${ }^{3}$, \\ Shunichi Ikeda ${ }^{3}$, Mitsuya Ishikawa ${ }^{3}$, Ken Ishitani ${ }^{4}$, Yasuo Hirai ${ }^{4}$, Hideo Matsui ${ }^{4}$, \\ Yasuhiro Matsumura ${ }^{1}$ \\ ${ }^{1}$ Division of Developmental Therapeutics, Research Center for Innovative Oncology, National Cancer Center Hospital East, Kashiwa, \\ Japan; ${ }^{2} \mathrm{R} \&$ D Department Unit 1, JSR Life Sciences Corporation, Tsukuba, Japan; ${ }^{3}$ Department of Gynecology, National Cancer \\ Center Hospital, Tokyo, Japan; ${ }^{4}$ Department of Obstetrics and Gynecology, Tokyo Women's Medical University, Tokyo, Japan. \\ Email: yhmatsum@east.ncc.go.jp
}

Received July $26^{\text {th }}, 2013$; revised August $24^{\text {th }}, 2013$; accepted September $1^{\text {st }}, 2013$

Copyright (C) 2013 Yoshikatsu Koga et al. This is an open access article distributed under the Creative Commons Attribution License, which permits unrestricted use, distribution, and reproduction in any medium, provided the original work is properly cited.

\begin{abstract}
A simple and non-invasive method for detecting endometrial cancer in women with abnormal uterine bleeding is required. For this purpose, we prepared immuno-magnetic beads conjugated with anti-human EpCAM rat monoclonal antibody $(\mathrm{mAb}$ ) for isolating exfoliated endometrial cells including endometrial cancer cells in vaginal discharge. The affinities of the anti-human EpCAM rat mAbs were analyzed by flow cytometry and immunocytochemistry and then magnetic beads were conjugated with the mAbs. The rate of retrieval of endometrial cells using the immuno-magnetic beads was calculated. Endometrial cells were isolated using the immuno-magnetic beads from the vaginal discharges of 22 patients with endometrial cancer and 16 non-malignant controls. The isolated cells were stained using endometrial cancer specific-mAbs and analyzed by flow cytometry and imaging cytometry. The immuno-magnetic beads conjugated with high-affinity mAb (clone 1456) appeared to have very low auto-fluorescence. Sufficient enrichment of Ep-CAMpositive cells using immuno-magnetic beads was observed in both simulation and clinical samples. The overall sensitivities of flow cytometry and imaging cytometry to detect endometrial cancer cells were $72.7 \%$ and $45.5 \%$, respectively. Meanwhile, the overall specificities of flow cytometry and imaging cytometry for healthy controls were $75.0 \%$ and $81.3 \%$, respectively. Our immuno-magnetic beads have very low auto-fluorescence, so they could be useful for fluorescent analysis, such as fluorescent immunochemical staining. In the future, these novel immuno-magnetic beads could be used for cytological study.
\end{abstract}

Keywords: Immuno-Magnetic Beads; Auto-Fluorescence; Endometrial Cancer; Cancer Screening

\section{Introduction}

Endometrial cancer is one of the most common malignancies of the female genital tract globally [1]. In the USA, approximately $80 \%$ of patients with cancer of the uterus were diagnosed as having endometrial cancer [2] and the number of patients with endometrial cancer has been increasing in Japan [3]. Regarding cancer screening of female genital cancers, cervical cancer of the uterus can be diagnosed more simply than endometrial cancer [4]. However, there is no screening method for endometrial cancer at present [5]. Abnormal uterine bleeding

*Disclosures: The authors declare that no actual or potential conflicts of interest exist. is a common symptom of endometrial cancer; however, endometrial cancer is diagnosed in only $10 \%$ of women with abnormal uterine bleeding [6,7] and no organic cause is found in $60 \%$ to $70 \%$ of these women [8]. For many years, dilatation followed by curettage has been the standard method for detecting endometrial cancer in women with abnormal uterine bleeding. In the last decade, several screening methods for women with abnormal uterine bleeding, such as transvaginal sonography [9,10], endometrial cytology [10], suction endometrial curettage [10], and endometrial sampling [10,11], were reported to reduce the cost and invasiveness. However, these screening methods are still invasive, time-con- 
suming, and expensive, and above all, inconvenient for women. Therefore, a simple, economic, and non-invasive method of detecting cancer through cancer screening for patients with abnormal uterine bleeding is required.

Recently, we reported a cytological analysis for the detection of endometrial cancer cells combined with endometrial cancer-specific monoclonal antibodies (mAbs) and imaging cytometry using exfoliated endometrial cells [12]. Exfoliated endometrial cancer cells and normal endometrial cells were observed in the vaginal discharge of patients with endometrial cancer. In addition, to detect endometrial cancer cells, mAbs against cysteine-rich with EGF-like domain 1 (CRELD1), G-protein-coupled receptor kinase 5 (GRK5), solute carrier family 25 member 27 (SLC25A27), and stanniocalcin 2 (STC2) were used. However, almost all tampon-retrieved cells were granulocytes and normal squamous cells from the vagina, and the rate of endometrial cells in all tampon-retrieved cells was less than $1 \%[12]$.

Meanwhile, to isolate exfoliated colonocytes from feces, we previously reported immuno-magnetic beads conjugated with anti-human epithelial cell adhesion molecule (EpCAM) mouse mAb (Magnosphere MC290/ anti-EpCAM mouse IgG, JSR Life Sciences, Tsukuba, Japan) [13]. The same as for fecal samples, exfoliated endometrial cells could be isolated using immuno-magnetic beads. The capturing mAbs (anti-human EpCAM mAbs conjugated to Magnosphere) and the detecting mAbs (endometrial cancer-specific mAbs) are both mouse monoclonal antibodies; thus, the secondary antibody for immunochemical staining cross-reacted with the $\mathrm{mAbs}$ for capture and those for detection. To resolve this issue, we have established anti-human EpCAM rat mAb and immuno-magnetic beads with very low auto-fluorescence for the isolation of exfoliated endometrial cells in the present study. Then, the exfoliated endometrial cells collected using immuno-magnetic beads were subjected to cellular analysis.

\section{Materials and Methods}

\subsection{Establishment of Anti-Human EpCAM Rat Monoclonal Antibodies}

A recombinant human EpCAM/Fc chimera (R \& D Systems, Minneapolis, MN) was used as an immunogen. 0.1 $\mathrm{mg}$ of the antigen was mixed with complete Freund's adjuvant (Difco, Detroit, MI) and injected intraperitoneally into Wistar rat (Japan SLC, Shizuoka, Japan). The ELISA-positive hybridoma cells were cloned by limiting dilution in 96-well culture plates and established as stable hybridoma cells.

Immunoglobulin $\mathrm{G}$ was separately purified from each ascites fluid sample using protein $\mathrm{G}$ affinity chromatog- raphy (GE Healthcare Life Science, Piscataway, NJ). The purified IgG fractions were used for further characterization and were evaluated for their reactivity.

\subsection{Flow Cytometry of Anti-Human EpCAM mAb}

HT-29 cells and UMUC-3 cells were used as EpCAMpositive and -negative cells, respectively. $2 \times 10^{5}$ trypsinized cells were put into a $2-\mathrm{mL}$ tube and incubated with $0.2 \mu \mathrm{g}$ of each $\mathrm{mAb}$ for $30 \mathrm{~min}$ at $4{ }^{\circ} \mathrm{C}$. After rinsing with PBS containing $0.5 \%$ bovine serum albumin (BSA) and $2 \mathrm{mM}$ ethylenediaminetetraacetic acid (EDTA) (B. E. PBS), the cells were incubated with $0.1 \mu \mathrm{g}$ of DyLight 649-conjugated donkey anti-rat IgG secondary antibody (Jackson ImmunoResearch, West Grove, PA) for $30 \mathrm{~min}$ at $4^{\circ} \mathrm{C}$. Finally, the cells were rinsed with B. E. PBS and nuclear-stained with propidium iodide (PI) solution (Invitrogen, Eugene, OR). The stained cells were analyzed by flow cytometry using a Guava easyCyte.

\subsection{Immunocytochemistry of Anti-Human EpCAM mAb}

EpCAM-positive and -negative cells were pre-cultured on BD Falcon culture slides (BD Biosciences, Bedford, MA). The cells were fixed in $4 \%$ paraformaldehyde (Wako, Osaka, Japan) for $30 \mathrm{~min}$ at $4^{\circ} \mathrm{C}$ and rinsed with ultrapure water. Endogenous peroxidase was blocked with a $3 \%$ hydrogen peroxide solution in $100 \%$ methanol for $30 \mathrm{~min}$ at room temperature, followed by rinsing with PBS. Nonspecific protein binding was blocked with 5\% skim milk in PBS for $30 \mathrm{~min}$ at room temperature. After draining off the skim milk solution, the cells were incubated with $4 \mu \mathrm{g}$ of each anti-human EpCAM rat mAb for $1 \mathrm{hr}$ at room temperature. After rinsing with PBS, the cells were incubated with $0.1 \mu \mathrm{g}$ of HRP-conjugated donkey anti-rat IgG secondary antibody (Jackson ImmunoResearch) for $30 \mathrm{~min}$ at room temperature. After rinsing with PBS, the cells were incubated with the 3,3'-diaminobenzidine tetrahydrochloride $(\mathrm{DAB}+)$ liquid system (Dako, Glostrup, Denmark) for 5 min at room temperature. Finally, the cells were rinsed and counter-stained with hematoxylin solution.

\subsection{Immuno-Magnetic Beads Conjugated with Anti-Human EpCAM Rat mAb}

Magnetic beads of $3.0 \mu \mathrm{m}$ diameter, Magnosphere MC290/Tosyl (JSL Life Sciences), were prepared in this study. The magnetic beads were directly conjugated with anti-human EpCAM rat mAb. The sizes of the obtained magnetic beads were determined to be $3.0 \mu \mathrm{m}$ based on electron microscopic observation. Also, commercially available immuno-magnetic beads conjugated with anti- 
human EpCAM mouse mAb, Dynabeads Epithelial Enrich (Dynal, Oslo, Norway) and Magnosphere MC290/ anti-EpCAM mouse IgG (JSR Life Sciences), were used in the present study.

\subsection{Simulation Analysis for Retrieving Cells Using Immuno-Magnetic Beads}

In the present study, the immuno-magnetic beads were used for the isolation of endometrial cells from the samples of abnormal uterine bleeding or menstrual bleeding; thus, EpCAM-positive cells added to peripheral blood were used in the simulation study. $1 \times 10^{6}$ HT-29 cells with $3 \mathrm{~mL}$ of peripheral blood (approximately $1 \times 10^{7}$ granulocytes) were prepared in the simulation study. 30 $\mathrm{mL}$ of a retrieval PBS buffer containing $0.1 \%$ BSA and 2 mM EDTA was added to the sample. The EpCAMpositive cells were captured using $40 \mu \mathrm{L}$ of immunomagnetic beads, and the mixtures were incubated for 30 min under gentle rolling at room temperature. Then, the mixtures on the magnet were incubated on a shaking platform for $15 \mathrm{~min}$ at room temperature. Subsequently, the supernatant was removed and the retrieved cells were fixed in $4 \%$ paraformaldehyde for $30 \mathrm{~min}$ at $4{ }^{\circ} \mathrm{C}$. In the clinical simulation study, menstrual blood from a healthy volunteer was subjected to the same method as described above.

To analyze the efficiency of immuno-magnetic beads, the cells retrieved using the immuno-magnetic beads were stained with the anti-human EpCAM goat polyclonal $\mathrm{Ab}$ (R \& D Systems) and assessed using an imaging cytometer, CELAVIEW (Olympus, Tokyo, Japan). The retrieved cells were plated into each well of flatbottomed 96-well tissue culture plates (Corning). The plates were centrifuged at $30 \mathrm{~g}$ for $5 \mathrm{~min}$ at $4^{\circ} \mathrm{C}$ and the supernatant was removed, followed by drying over-night at room temperature. The cells were permeabilized by incubation in PBS containing $0.2 \%$ Triton X-100 for 20 $\mathrm{min}$ at room temperature. Non-specific protein binding was blocked with 5\% skim milk in PBS for $30 \mathrm{~min}$ at room temperature. After draining off the skim milk solution, the cells were incubated with $1 \mu \mathrm{g}$ of commercially available anti-human EpCAM goat polyclonal $\mathrm{Ab}$, followed by incubation for $1 \mathrm{hr}$ at room temperature. After rinsing with PBS, the sections were incubated with 0.1 $\mu \mathrm{g}$ of DyLight 488-conjugated bovine anti-goat IgG secondary antibody (Jackson ImmunoResearch) for $30 \mathrm{~min}$ at room temperature.

\subsection{Patients with Endometrial Cancer or Non-Malignant Controls}

From January 2012 to December 2012, 22 patients with histologically confirmed endometrial cancer and 16 non- malignant controls before menopause were enrolled in this study (Table 1). All the patients had undergone surgical resection of their primary cancer at the National Cancer Center Hospital, Tokyo, Japan. The endometrial cancer patients were slightly older than the non-malignant controls. All patients and controls received detailed information about the study and gave written consent to participate in the study, which was approved by the Institutional Review Board of the National Cancer Center, Japan, and Tokyo Women's Medical University.

\subsection{Retrieval of Naturally Exfoliated Endometrial Cells Using Immuno-Magnetic Beads}

The participants in this study inserted a small tampon (3 $\times 1 \mathrm{~cm}$, Unicharm, Tokyo, Japan) into their vagina and took the tampon out after about 3 hrs [12]. The tampon was then placed in a centrifuge tube (Corning) containing $30 \mathrm{~mL}$ of retrieval buffer and was shipped immediately to our laboratory at $4^{\circ} \mathrm{C}$. To retrieve the exfoliated cells from the tampon, it was pressed thoroughly and all

Table 1. Characteristics of endometrial cancer patients and non-malignant controls.

\begin{tabular}{|c|c|c|}
\hline & Patients $(\mathrm{N}=22)$ & Controls $(\mathrm{N}=16$ \\
\hline Age [median (range)] & $60(47-75)$ & $41(29-52)$ \\
\hline $\begin{array}{c}\text { EpCAM + cells FCM } \\
\text { [median (range)] }\end{array}$ & $17(1-2322)$ & $113(2-1964)$ \\
\hline $\begin{array}{l}\text { EpCAM + cells ICM } \\
{[\text { median (range) }]}\end{array}$ & $17(0-1893)$ & $82(0-2014)$ \\
\hline \multicolumn{3}{|l|}{ Histology [Number (\%)] } \\
\hline $\begin{array}{c}\text { Endometrioid } \\
\text { adenocarcinoma }\end{array}$ & $18(81.8 \%)$ & \\
\hline Mixed adenocarcinoma & $2(9.1 \%)$ & \\
\hline Serous adenocarcinoma & $1(4.5 \%)$ & \\
\hline Carcinosarcoma & $1(4.5 \%)$ & \\
\hline \multicolumn{3}{|l|}{$\begin{array}{l}\text { FIGO classification } \\
\text { [Number (\%)] }\end{array}$} \\
\hline stage I & $14(63.6 \%)$ & \\
\hline stage II & $4(18.2 \%)$ & \\
\hline stage III & $3(13.6 \%)$ & \\
\hline stage IV & $1(4.5 \%)$ & \\
\hline $\begin{array}{c}\text { Tumor size } \\
{[\mathrm{mm}, \text { median (range) }]}\end{array}$ & $37(9-82)$ & \\
\hline
\end{tabular}

Patients: patients with endometrial cancer, Controls: non-malignant patients without malignancy in the uterus, EpCAM + cells FCM: positively stained cells using EpCAM Ab counted by flow cytometry, EpCAM + cells ICM: positively stained cells using EpCAM Ab counted by imaging cytometry, FIGO: International Federation of Gynecology and Obstetrics. 
obtained fluid was collected in another new centrifuge tube. The exfoliated endometrial cells were captured by adding $40 \mu \mathrm{L}$ of immuno-magnetic beads into the sample solution, and the mixtures were incubated for $30 \mathrm{~min}$ under gentle rolling conditions at room temperature. The mixtures on the magnet were incubated on a shaking platform for $15 \mathrm{~min}$ at room temperature. Then, the supernatant was removed and the retrieved cells were fixed in $4 \%$ paraformaldehyde for $30 \mathrm{~min}$ at $4^{\circ} \mathrm{C}$. The cells were rinsed with PBS and stored in $1 \mathrm{~mL}$ of PBSbased storage buffer containing $0.1 \%$ BSA, 2 mM EDTA, and $0.1 \% \mathrm{NaN} 3$ at $4^{\circ} \mathrm{C}$ until analysis.

\subsection{Cellular Analysis Using Flow Cytometry and Imaging Cytometry}

For cellular analysis using flow cytometry, the exfoliated endometrial cells retrieved using the immuno-magnetic beads were put into a $2-\mathrm{mL}$ tube and incubated with $4 \mu \mathrm{g}$ of anti-CRELD1 (clone 2D1E12I), GRK5 (clone 2F11C3), SLC25A27 (clone 3A8B14), and STC2 (clone 2D4C4) mouse mAbs (ACTGen, Nagano, Japan) as well as $1 \mu \mathrm{g}$ of anti-human EpCAM goat polyclonal antibody for $1 \mathrm{hr}$ at room temperature. After rinsing with retrieval buffer, the cells were incubated with $0.1 \mu \mathrm{g}$ of DyLight 488conjugated bovine anti-goat IgG secondary antibody and $0.1 \mu \mathrm{g}$ of DyLight 649-conjugated donkey anti-mouse IgG secondary antibody for $30 \mathrm{~min}$ at room temperature. Finally, the cells were rinsed with retrieval buffer and nuclear-stained with PI solution. The stained cells were analyzed using flow cytometry with a Guava easyCyte (Millipore, Billerica, MA).

For cellular analysis using imaging cytometry, the exfoliated endometrial cells retrieved using immunomagnetic beads were prepared as described in the simulation experiment.

\section{Results}

\subsection{Evaluation of Anti-Human EpCAM Rat Monoclonal Antibodies}

Mean intensities of EpCAM positive cells with isotype control (negative control), clone B8-4 (positive control), clone 118 , clone 533, clone 572, clone 787, clone 1097, clone 1286 , clone 1456 , and clone 1468 were $3.33,1683$, $1823,810,1865,1693,1571,1531,1670$, and 861 , respectively (Figure 1(a)). Mean intensities of EpCAMnegative cells with the same mAbs were $3.54,3.75,5.00$, $3.52,3.92,12.9,5.41,6.33,3.58$, and 3.24 , respectively (Figure 1(b)). The affinities to EpCAM-positive cells using two mAbs (clones 533 and 1468) were lower than that to clone B8-4. Meanwhile, non-specific reactions to EpCAM-negative cells were observed for four mAbs (clones 118, 787, 1097, and 1286). From the results of flow cytometry, two mAbs (clones 572 and 1456) were selected as candidates for the conjugation. In the immunocytochemical staining, EpCAM-positive cells were stained positively by clone B8-4 and clone $1456 \mathrm{mAbs}$ (Figure 1(c)). However, these cells could not be stained by clone $572 \mathrm{mAb}$. Thus, clone 1456 was used as antihuman EpCAM rat mAb in the following experiments.

\subsection{Low Auto-Fluorescence of Immuno-Magnetic Beads Conjugated with Anti-Human EpCAM Rat mAb}

The levels of auto-fluorescence of commercially available immuno-magnetic beads (Dynabeads Epithelial Enrich and MC290/EpCAM mouse IgG) and our new immuno-magnetic beads (MC290/EpCAM rat IgG) were compared (Figure 2(a)). Fluorescent intensities were assessed at wavelengths of excitation (Ex) $488 \mathrm{~nm} / \mathrm{emis}-$ sion (Em) $525 \mathrm{~nm}$ (green fluorescence), Ex $488 \mathrm{~nm} / \mathrm{Em}$ $583 \mathrm{~nm}$ (yellow fluorescence), Ex $488 \mathrm{~nm} / \mathrm{Em} 680 \mathrm{~nm}$ (red fluorescence), and Ex $640 \mathrm{~nm} / \mathrm{Em} 661 \mathrm{~nm}$ (red2 fluorescence). Mean intensities of Dynabeads Epithelial Enrich in terms of green, yellow, red, and red2 fluorescence were $13.7,41.5,44.3$, and 10.8 , respectively. Corresponding mean intensities of MC290/EpCAM mouse $\mathrm{IgG}$ were $3.01,4.61,4.05$, and 6.03 , respectively. In addition, mean intensities of MC290/EpCAM rat IgG were $2.82,4.20,3.29$, and 6.35 , respectively. Consequently, MC290 beads showed low auto-fluorescence and Dynabeads showed high auto-fluorescence. The binding of Dynabeads and MC290 beads to EpCAM-positive cells

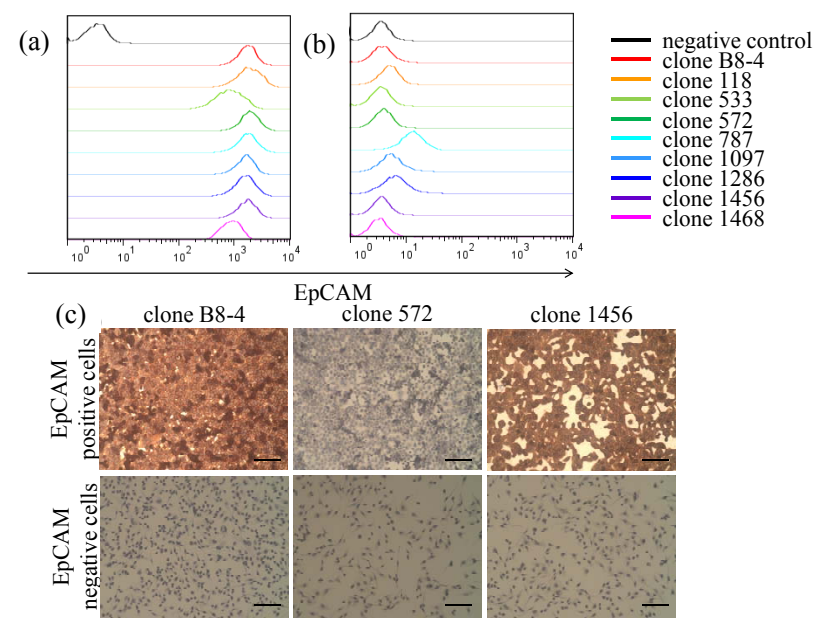

Figure 1. Evaluation of anti-human EpCAM rat monoclonal antibodies. (a) Flow cytometry using EpCAM-positive cells. Seven clones of anti-human EpCAM rat mAbs were compared to a positive control (anti-human EpCAM mouse mAb; clone B8-4). (b) Flow cytometry using EpCAM-negative cells. (c) Immunocytochemistry of EpCAMpositive and -negative cells using clone B8-4, clone 1456, and clone 572. Scale bar represents $100 \mu \mathrm{m}$. 
is shown in Figure 2(b). EpCAM-positive cells were retrieved by using both Dynabeads Epithelial Enrich and MC290 beads; however, Dynabeads showed auto-fluorescence. Meanwhile, MC290 had the lowest level of auto-fluorescence. Thus, our MC290/EpCAM rat IgG appeared to be useful for fluorescent immunochemistry.

\subsection{Cell Isolation Using Immuno-Magnetic Beads Conjugated with Anti-Human EpCAM Rat mAb}

In the simulation experiment, EpCAM-positive cells were added to granulocytes at a ratio of approximately $1: 10$. The actual proportion of EpCAM-positive cells was 8.8\% (EpCAM-positive cells/all cells: 659/7508) (Figures 3(a) and (e)). After the isolation using the beads, the rate of EpCAM-positive cells was 48.5\% (306/634) (Figures 3(b) and (f)). In the clinical sample, menstrual blood of a healthy volunteer was used. The rate of EpCAM-positive cells in the clinical subject was 3.0\% $(361 / 12,112)$ (Figures 3(c) and (g)). After the isolation using the beads, the rate of EpCAM-positive cells in the clinical subject was 61.9\% (224/394) (Figures 3(d) and (h)). Recovery rate of EpCAM-positive cells using immuno-magnetic beads was $50 \%$ to $67 \%$. Thus, EpCAM-positive cells before cell isolation were more than that after cell isolation. All cells were stained by DAPI (blue) and EpCAM-positive cells were stained by anti-EpCAM mAb (green) and DAPI (blue). After cell isolation, many particles without staining as shown in the Figures 3(f) and (h) were immuno-magnetic beads. Sufficient enrichment of EpCAMpositive cells using MC290/EpCAM rat IgG was thus observed in both the simulation experiment and the clinical samples.

\subsection{Clinical Evaluation of Cellular Analysis}

The median numbers of EpCAM-positive cells observed in endometrial cancer patients using flow cytometry and imaging cytometry were 17 (range 1 - 2322) and 17 (0 1893), respectively. Meanwhile, the median numbers of EpCAM-positive cells observed in healthy controls using flow cytometry and imaging cytometry were 113 (range 2 - 1964) and 82 (0 - 2014), respectively. The samples with less than 10 EpCAM-positive cells were not applicable in this study. Samples with over 10 EpCAM-positive cells were examined and for practical purpose, more than $20 \%$ positive by the cancer detecting mAbs were designated "positive". The sensitivities and specificities of cellular analyses using flow cytometry and imaging cytometry are shown in Table 2. In the flow cytometry, immuno-staining-positive cells using CRELD1, GRK5, SLC25A27, and STC2 mAbs were observed in $40.9 \%$, $18.2 \%, 18.2 \%$, and $27.3 \%$ of endometrial cancer patients,

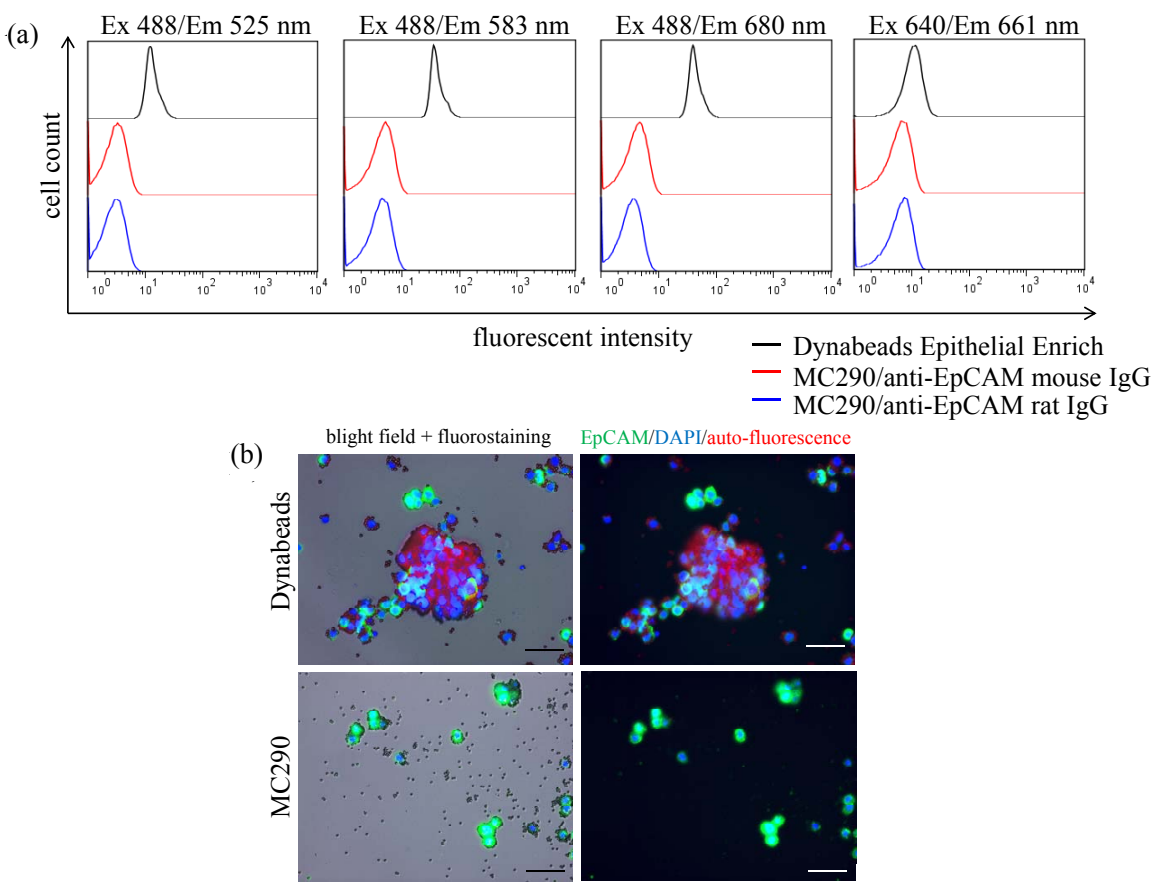

Figure 2. Auto-fluorescence of each bead. (a) Auto-fluorescence of Dynabeads Epithelial Enrich, MC290/EpCAM mouse IgG and MC290/EpCAM rat IgG. Fluorescent intensities were assessed at wavelengths of excitation (Ex) $488 \mathrm{~nm} / \mathrm{emission}(\mathrm{Em})$ $525 \mathrm{~nm}, \operatorname{Ex} 488 \mathrm{~nm} / \mathrm{Em} 583 \mathrm{~nm}, \operatorname{Ex} 488 \mathrm{~nm} / \mathrm{Em} 680 \mathrm{~nm}$, and Ex $640 \mathrm{~nm} / \mathrm{Em} 661 \mathrm{~nm}$. (b) Binding abilities of Dynabeads and MC290 beads to EpCAM-positive cells. EpCAM-positive cells were retrieved by using both Dynabeads Epithelial Enrich and MC290 beads. Dynabeads exhibited some auto-fluorescence, while MC290 exhibited less. Scale bar represents $100 \mu \mathrm{m}$. 


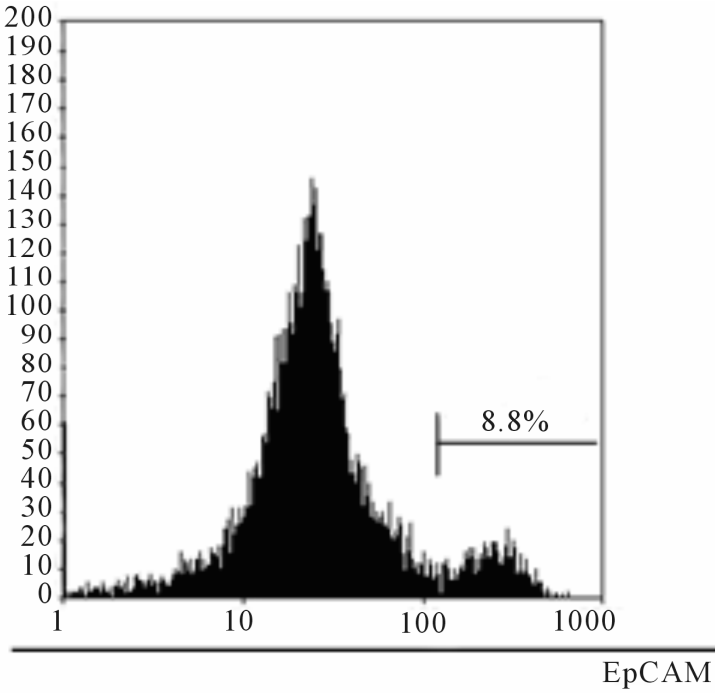

(a)

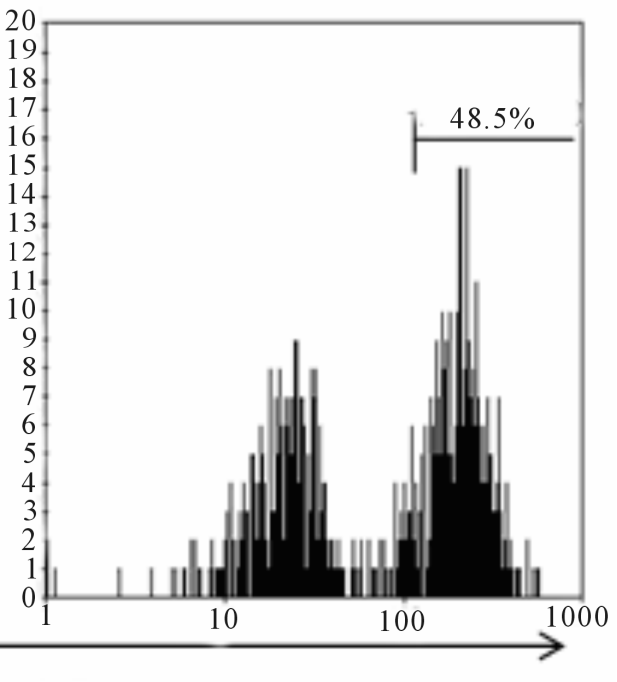

(b)

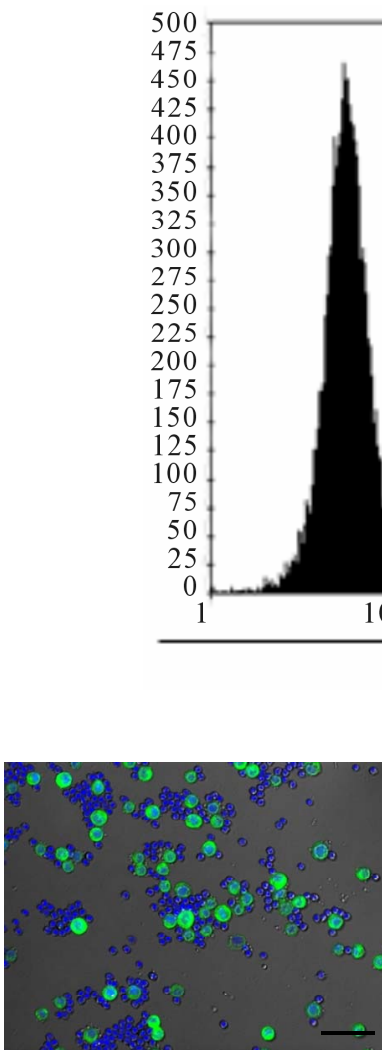

(e)

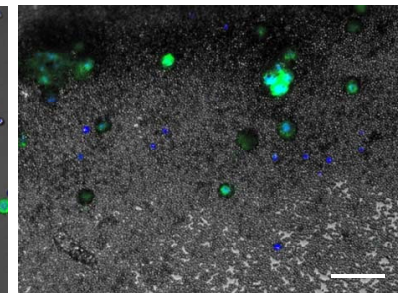

(f)

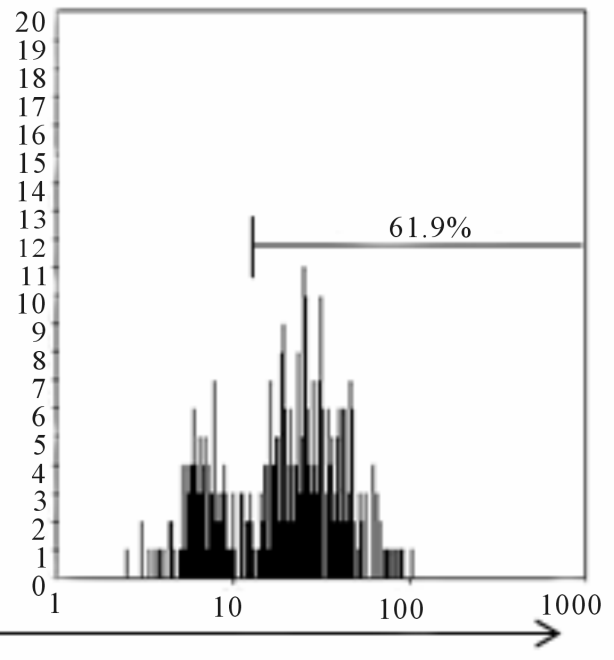

(d)

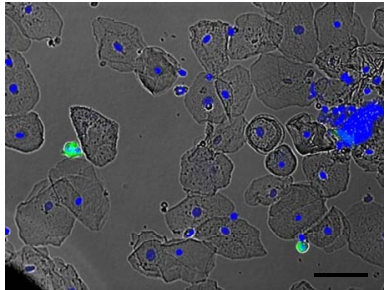

(g)

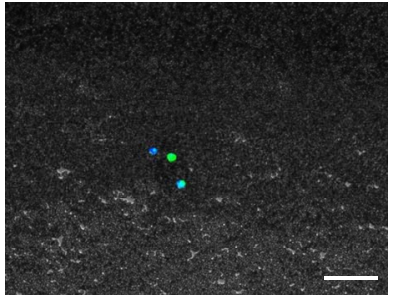

(h)

Figure 3. Cell isolation using immuno-magnetic beads tagged with anti-human EpCAM rat mAb. (a) Imaging cytometry of simulation study before cell isolation. The rate of EpCAM-positive cells in the simulation subject before bead isolation was 8.8\% (EpCAM-positive cells/all cells: 659/7508). (b) Imaging cytometry of simulation study after cell isolation. The rate of EpCAM-positive cells in the simulation subject after bead isolation was $48.5 \%(306 / 634)$. (c) Imaging cytometry of clinical simulation study before cell isolation. The rate of EpCAM-positive cells in the clinical subject before bead isolation was $3.0 \%$ $(361 / 12,112)$. (d) Imaging cytometry of clinical simulation study after cell isolation. The rate of EpCAM-positive cells in the clinical subject after bead isolation was $61.9 \%(224 / 394)$. (e) Immunocytochemistry of simulation study before cell isolation. (f) Immunocytochemistry of simulation study after cell isolation. (g) Immunocytochemistry of clinical simulation study before cell isolation. (h) Immunocytochemistry of clinical simulation study after cell isolation. EpCAM protein was stained with DyLight 488 (green) and the nucleus was stained with DAPI (blue). Scale bar represents $100 \mu \mathrm{m}$. 
Table 2. Sensitivities and specificities of cellular analyses using flow cytometry and imaging cytometry.

\begin{tabular}{|c|c|c|c|c|}
\hline & \multicolumn{4}{|c|}{ Endometrial cancer patients $(\mathrm{N}=22)$} \\
\hline & \multicolumn{2}{|r|}{ Flow cytometry } & \multicolumn{2}{|c|}{ Imaging cytometry } \\
\hline & No. & Sensitivity $(\%, 95 \% \mathrm{CI})$ & No. & Sensitivity $(\%, 95 \% \mathrm{CI})$ \\
\hline Combined marker & 16 & $72.7 \%(49.8-89.3)$ & 10 & $45.5 \%(24.4-67.8)$ \\
\hline CRELD1 & 9 & $40.9 \%(20.7-63.7)$ & 4 & $18.2 \%(5.2-40.3)$ \\
\hline GRK5 & 4 & $18.2 \%(5.2-40.3)$ & 4 & $18.2 \%(5.2-40.3)$ \\
\hline SLC25A27 & 4 & $18.2 \%(5.2-40.3)$ & 2 & $9.1 \%(1.1-29.2)$ \\
\hline \multirow[t]{4}{*}{ STC2 } & 6 & $27.3 \%(10.7-50.3)$ & 2 & $9.1 \%(1.1-29.2)$ \\
\hline & \multicolumn{4}{|c|}{ Non-malignant controls $(\mathrm{N}=16)$} \\
\hline & \multicolumn{2}{|r|}{ Flow cytometry } & \multicolumn{2}{|c|}{ Imaging cytometry } \\
\hline & No. & Specificity $(\%, 95 \% \mathrm{CI})$ & No. & Specificity $(\%, 95 \% \mathrm{CI})$ \\
\hline Combined marker & 12 & $75.0 \%(47.7-92.7)$ & 13 & $81.3 \%(54.3-96.0)$ \\
\hline CRELD1 & 14 & $87.5 \%(61.7-98.5)$ & 15 & $93.8 \%(69.8-99.8)$ \\
\hline GRK5 & 15 & $93.8 \%(69.8-99.8)$ & 16 & $100 \%(79.4-100)$ \\
\hline SLC25A27 & 14 & $87.5 \%(61.7-98.5)$ & 15 & $93.8 \%(69.8-99.8)$ \\
\hline STC2 & 15 & $93.8 \%(69.8-99.8)$ & 14 & $87.5 \%(61.7-98.5)$ \\
\hline
\end{tabular}

CRELD1: cysteine-rich with EGF-like domain 1 (clone 2D1E12I), GRK5: G-protein-coupled receptor kinase 5 (clone 2F11C3), SLC25A27: solute carrier family 25 member 27 (clone 3A8B14), STC2: stanniocalcin 2 (clone 2D4C4), 95\% CI: 95\% confidence interval.

respectively. In the imaging cytometry, immuno-staining-positive cells using CRELD1, GRK5, SLC25A27, and STC2 mAbs were observed in $18.2 \%, 18.2 \%, 9.1 \%$, and $9.1 \%$ of endometrial cancer patients, respectively. Approximately $90 \%$ of non-malignant controls were negative in both flow cytometry and imaging cytometry using these cancer-specific mAbs. The overall sensitivities of flow cytometry and imaging cytometry to detect endometrial cancer cells were $72.7 \%$ [95\% confidence interval (CI), 49.8 - 89.3] and 45.5\% (95\% CI, 24.4 67.8 ), respectively. On the other hand, the overall specificities of flow cytometry and imaging cytometry for non-malignant controls were $75.0 \%$ (95\% CI, 47.7 - 92.7) and $81.3 \%(95 \% \mathrm{CI}, 54.3-96.0)$, respectively.

\section{Discussion}

The tampon retrieval sample contained several kinds of exfoliated cell, such as granulocytes, normal squamous cells, and endometrial cells [12]. Thus, a cell isolation method using immuno-magnetic beads was important for the isolation of exfoliated endometrial cells from tampon. There were two problems regarding the fluorescent immunochemical staining of exfoliated endometrial cells retrieved using immuno-magnetic beads. One was that the fluorescent secondary antibody cross-reacted to both the capturing $\mathrm{mAb}$ and the detecting $\mathrm{mAb}$ because both were mouse mAbs. To resolve this issue, we succeeded in developing anti-human EpCAM rat IgG (clone 1456), the affinity of which was as high as that of anti-human EpCAM mouse IgG (clone B8-4). We established clone B8-4 as high affinity $\mathrm{mAb}$ in the previous study [13]. Clone B8-4 was useful for immunohistochemistry, flow cytometry, and cell isolation. Thus, we used clone B8-4 as a positive control in this study. We think that clone 572 may recognize protein structure of EpCAM antigen on living cells, thus, this mAb was positive for flow cytometry but negative for immunochemical staining. The other problem to be resolved was the auto-fluorescence of immuno-magnetic beads. The fluorescent intensity cannot be calculated correctly if the immuno-magnetic beads exhibit auto-fluorescence. Polyurethanes are frequently used in biomedical applications because of their excellent biocompatibility $[14,15]$. However, polyurethanes are known to emit auto-fluorescence [16]. Be-cause the commercially available immuno-magnetic beads (Dynabeads Epithelial Enrich) are coated with polyurethane emitting auto-fluorescence, these beads cannot be used in our cellular analysis. The beads prepared by us exhibited very low auto-fluorescence because the surfaces of our immuno-magnetic beads are coated with a poly-glycidyl methacrylate layer that does not emit autofluorescence. 
In the simulation study, our newly developed immunomagnetic beads could retrieve EpCAM-positive cells efficiently. Moreover, the beads could isolate the endometrial cells from various types of cell adsorbed to a tampon. Both endometrial cancer cells and normal endometrial cells were EpCAM-positive. Almost EpCAMpositive cells from non-malignant controls were exfoliated normal endometrial cells. Meanwhile, EpCAMpositive cells from endometrial cancers were consisted of endometrial cancer cells and normal endometrial cells. Not only endometrial cancer cells but also tubal cancer, cervical cancer, or vaginal cancer cells may be exfoliated into a vaginal discharge. There is, therefore, potential to detect these cells in a same sample. In this study, magnetic beads conjugated with anti-human EpCAM mAb (EpCAM-beads) were used for isolation of exfoliated endometrial cancer cells from vaginal discharge. Since exfoliated cancer cells from squamous cell carcinoma (most of cervical cancer and vaginal cancer) are EpCAMnegative, they were not isolated by EpCAM-beads. On the other hand, exfoliated cancer cells from adenocarcinoma (most of tubal cancer and a few of cervical cancer) were isolated by EpCAM-beads because they were EpCAM-positive. Unfortunately, we do not have a $\mathrm{mAb}$ which can distinguish cervical cancer cells from normal squamous cells. In our previous study, most tamponretrieved cells were granulocytes and normal squamous cells [12] and the rate of endometrial cells in menstrual blood was only $3 \%$ in this study. In particular, few endometrial cells were obtained from among tampon-retrieved cells of the non-malignant control in a non-menstrual period. The major symptom of endometrial cancer is abnormal uterine bleeding; thus, subjects with abnormal uterine bleeding or normal menstrual blood were enrolled in this clinical study as controls. A lot of EpCAM-positive endometrial cells were obtained from controls with menstrual period but the least EpCAMpositive cells were obtained from controls with non-menstrual period. Thus, menstrual blood was used for nonmalignant control in this study. The median EpCAMpositive cells of controls were over 100. Meanwhile, major symptom of endometrial cancer patient was abnormal uterine bleeding. However, almost patients with endometrial cancer were in menopause and their endometria had changed to be atrophic. Therefore, a few exfoliated EpCAM-positive cells were obtained from patients with endometrial cancer but most of them appeared to be malignant endometrial cells. Immunomagnetic beads that could retrieve the endometrial cells from a sample of uterine blood appeared to be useful.

In this study, the detecting mAbs were the same as in our previous study [12]. Recently, several mAbs against EpCAM, EphA2, MMP2, survivin, and podoplanin were investigated for endometrial cancer treatment and the detection of lymphovascular invasion [15,17-19]. However, these mAbs insufficient to detect endometrial cancer cells, so further development of endometrial cancerspecific mAbs should be performed. Meanwhile, several microRNAs (miRNAs) that were highly expressed in endometrial cancer tissue were reported [20-22]. The miRNA expression test of exfoliated endometrial cells might become an alternative endometrial cancer screening method in the future.

The present study showed that the sensitivity to detect endometrial cancer patients and the specificity for nonmalignant controls with flow cytometry analysis were $73 \%$ and $75 \%$, respectively. Meanwhile, the sensitivity and the specificity by imaging cytometry analysis were $46 \%$ and $81 \%$, respectively. Generally, cancer screening is performed for average-risk population to reduce the cancer mortality in the group. Thus, cancer screening is performed annually and should be low-cost and non(less)-invasive. Although sufficient sensitivity of cancer screening is $50 \%$ to $75 \%$, specificity is needed over $95 \%$. In this context, we have to admit that specificity of our cellular analysis was low. We are, therefore, developing further specific $\mathrm{mAbs}$ to increase the specificity. Recently serum human epididymis protein 4 (HE4) has been used for the detection of endometrial cancer [23-27]. Angioli et al. reported that the sensitivity and specificity of serum HE4 test were $59 \%$ and $100 \%$, respectively [27]. Serum (plasma) protein analyses (containing HE4, CEA or CA125) were useful for detection of cancer relapse or therapeutic effect of chemotherapy. Therefore, these are used for so-called tumor markers as other cancers. For example, in colorectal cancer, CEA is useless for the early stage but is available as a tumor marker because serum CEA level in patients with early stage of colorectal cancer is not higher than that in healthy controls. Therefore, fecal occult blood test is generally used in colorectal cancer screening. With the same reason, serum protein was not useful for endometrial cancer screening and proteins derived from endometrial cancer cells were contained more in a vaginal discharge. It is then speculated that the vaginal discharge containing exfoliated cells is useful for endometrial cancer screening. These findings are still insufficient for endometrial cancer screening, so further investigations are needed.

Sensitivity and specificity of our cellular analysis are less than those of traditional methods, such as curettage. Histological diagnosis using endometrial cytology or biopsy is used for final diagnosis. However, examinees suffer physical and psychological pain with curettage. In addition, current screening methods including curettage are time consuming and expensive and the medical examination is inconvenient for women. Our novel cyto- 
logical or histological methods are non-invasive compared to former cytology or histology. Cellualr analysis using immuno-magnetic beads and fluorostaining could be performed without human's eye; thus, it could reduce the cost. Moreover, the procedures of cellular analysis can be performed automatically using immuno-magnetic beads and magnet. In this method, the women only collect vaginal discharge using a tampon and send the sample to a laboratory at suitable storage condition. Although both the sensitivity and specificity should be enhanced, we think that this method may become convenient and cheap, and the new mAbs sets detecting endometrial cancer should be developed. In this study, the number of clinical samples was small. Thus, many issues remain to be resolved in order to improve these cellular analyses for the detection of endometrial cancer cells using immuno-magnetic beads and flow cytometry. Our immunomagnetic beads have very low auto-fluorescence, so they should be useful for fluorescent analysis, such as fluorescent immunochemical staining. In the future, these novel materials could lead to the next-generation methodology for cellular diagnosis.

\section{Acknowledgements}

We thank Ms. Noriko Abe, Ms. Masae Ohmaru, Ms. Miyuki Miura and Ms. Yuka Nishina for their technical assistance and Ms. Kaoru Shiina for her secretarial assistance.

This work was supported by the Advanced Research for Medical Products Mining Programme of the National Institute of Biomedical Innovation (NIBIO) of Japan (Y. Koga); the Innovation Promotion Program from the New Energy and Industrial Technology Development Organization (NEDO) of Japan (Y. Matsumura); the Japan Society for the Promotion of Science (JSPS) through the Funding Program for World-Leading Innovative R \& D on Science and Technology (FIRST Program), initiated by the Council for Science and Technology Policy (CSTP) (Y. Matsumura); and the National Cancer Center Research and Development Fund (Y. Matsumura).

\section{REFERENCES}

[1] F. Amant, P. Moerman, P. Neven, D. Timmerman, E. Van Limbergen and I. Vergote, "Endometrial Cancer," Lancet, Vol. 366, No. 9484, 2005, pp. 491-505. http://dx.doi.org/10.1016/S0140-6736(05)67063-8

[2] R. Siegel, D. Naishadham and A. Jemal, "Cancer Statistics, 2013," CA: A Cancer Journal for Clinicians, Vol. 63, No. 1, 2013, pp. 11-30. http://dx.doi.org/10.3322/caac.21166

[3] T. Matsuda, T. Marugame, K. Kamo, K. Katanoda, W. Ajiki, T. Sobue and Group Japan Cancer Surveillance
Research, "Cancer Incidence and Incidence Rates in Japan in 2006: Based on Data from 15 Population-Based Cancer Registries in the Monitoring of Cancer Incidence in Japan (MCIJ) Project," Japanese Journal of Clinical Oncology, Vol. 42, No. 2, 2012, pp. 139-147. http://dx.doi.org/10.1093/jjco/hyr184

[4] R. A. Smith, D. Brooks, V. Cokkinides, D. Saslow and O. W. Brawley, "Cancer Screening in the United States, 2013: A Review of Current American Cancer Society Guidelines, Current Issues in Cancer Screening, and New Guidance on Cervical Cancer Screening and Lung Cancer Screening," CA: A Cancer Journal for Clinicians, Vol. 63, No. 2, 2013, pp. 88-105.

http://dx.doi.org/10.3322/caac.21174

[5] R. A. Smith, V. Cokkinides and O. W. Brawley, "Cancer Screening in the United States, 2009: A Review of Current American Cancer Society Guidelines and Issues in Cancer Screening," CA: A Cancer Journal for Clinicians, Vol. 59, No. 1, 2009, pp. 27-41. http://dx.doi.org/10.3322/caac.20008

[6] J. Holst, O. Koskela and B. von Schoultz, "Endometrial Findings Following Curettage in 2018 Women According to Age and Indications," Annales Chirurgiae et Gynaecologiae, Vol. 72, No. 5, 1983, pp. 274-277.

[7] T. Gredmark, S. Kvint, G. Havel and L. A. Mattsson, "Histopathological Findings in Women with Postmenopausal Bleeding," British Journal of Obstetrics and Gynaecology, Vol. 102, No. 2, 1995, pp. 133-136. http://dx.doi.org/10.1111/j.1471-0528.1995.tb09066.x

[8] Y. C. Choo, K. C. Mak, C. Hsu, T. S. Wong and H. K. $\mathrm{Ma}$, "Postmenopausal Uterine Bleeding of Nonorganic Cause," Obstetrics and Gynecology, Vol. 66, No. 2, 1985, pp. 225-228.

[9] B. Gull, B. Karlsson, I. Milsom and S. Granberg, "Can Ultrasound Replace Dilation and Curettage? A Longitudinal Evaluation of Postmenopausal Bleeding and Transvaginal Sonographic Measurement of the Endometrium as Predictors of Endometrial Cancer," American Journal of Obstetrics and Gynecology, Vol. 188, No. 2, 2003, pp. 401-408.

[10] E. Kondo, T. Tabata, Y. Koduka, K. Nishiura, K. Tanida, T. Okugawa and N. Sagawa, "What Is the Best Method of Detecting Endometrial Cancer in Outpatients? Endometrial Sampling, Suction Curettage, Endometrial Cytology," Cytopathology, Vol. 19, No. 1, 2008, pp. 28-33. http://dx.doi.org/10.1111/i.1365-2303.2007.00509.x

[11] B. R. Kipp, F. Medeiros, M. B. Campion, T. J. Distad, L. M. Peterson, G. L. Keeney, K. C. Halling and A. C. Clayton, "Direct Uterine Sampling with the Tao Brush Sampler Using a Liquid-Based Preparation Method for the Detection of Endometrial Cancer and Atypical Hyperplasia: A Feasibility Study," Cancer, Vol. 114, No. 4, 2008, pp. 228-235. http://dx.doi.org/10.1002/cncr.23636

[12] Y. Koga, M. Yasunaga, M. Kajikawa, E. Shimizu, R. Takamatsu, R. Kataoka, Y. Murase, Y. Sasajima, T. Kasamatsu, T. Kato, T. Onda, S. Ikeda, M. Ishikawa, K. Ishitani, H. Ohta and Y. Matsumura, "Novel Virtual Cytological Analysis for the Detection of Endometrial Can- 
cer Cells Using Autoscan Fluoromicroscopy," Cancer Science, Vol. 102, No. 5, 2011, pp. 1068-1075. http://dx.doi.org/10.1111/j.1349-7006.2011.01903.x

[13] Y. Koga, M. Yasunaga, S. Katayose, Y. Moriya, T. Akasu, S. Fujita, S. Yamamoto, H. Baba and Y. Matsumura, "Improved Recovery of Exfoliated Colonocytes from Feces Using Newly Developed Immunomagnetic Beads," Gastroenterology Research \& Practice, Vol. 2008, 2008, Article ID: 605273. http://dx.doi.org/10.1155/2008/605273

[14] S. Grenier, M. Sandig, D. W. Holdsworth and K. Mequanint, "Interactions of Coronary Artery Smooth Muscle Cells with 3D Porous Polyurethane Scaffolds," Journal of Biomedical Materials Research Part A, Vol. 89, No. 2, 2009, pp. 293-303. http://dx.doi.org/10.1002/jbm.a.31972

[15] W. M. Merritt, A. A. Kamat, J. Y. Hwang, J. BottsfordMiller, C. Lu, Y. G. Lin, D. Coffey, W. A. Spannuth, E. Nugent, L. Y. Han, C. N. Landen, A. M. Nick, R. L. Stone, K. Coffman, E. Bruckheimer, R. R. Broaddus, D. M. Gershenson, R. L. Coleman and A. K. Sood, "Clinical and Biological Impact of EphA2 Overexpression and Angiogenesis in Endometrial Cancer," Cancer Biological Therapy, Vol. 10, No. 12, 2010, pp. 1306-1314. http://dx.doi.org/10.4161/cbt.10.12.13582

[16] S.-K. Wang and C. S. P. Sung, "Spectroscopic Characterization of Model Urea, Urethane Compound, and Diamine Extender for Polyurethane-Urea," Macromolecules, Vol. 35, No. 3, 2002, pp. 877-882. http://dx.doi.org/10.1021/ma011316+

[17] K. El-Sahwi, S. Bellone, E. Cocco, F. Casagrande, M. Bellone, M. Abu-Khalaf, N. Buza, F. A. Tavassoli, P. Hui, D. Ruttinger, D. A. Silasi, M. Azodi, P. E. Schwartz, T. J. Rutherford, S. Pecorelli and A. D. Santin, "Overexpression of EpCAM in Uterine Serous Papillary Carcinoma: Implications for EpCAM-Specific Immunotherapy with Human Monoclonal Antibody Adecatumumab (MT201)," Molecular Cancer Therapy, Vol. 9, No. 1, 2010, pp. 5766. http://dx.doi.org/10.1158/1535-7163.MCT-09-0675

[18] E. Yilmaz, M. Koyuncuoglu, I. B. Gorken, E. Okyay, B. Saatli, E. C. Ulukus and U. Saygili, "Expression of Matrix Metalloproteinase-2 and Survivin in Endometrioid and Nonendometrioid Endometrial Cancers and Clinicopathologic Significance," Journal of Gynecologic Oncology, Vol. 22, No. 2, 2011, pp. 89-96. http://dx.doi.org/10.3802/jgo.2011.22.2.89

[19] S. K. Weber, A. Sauerwald, M. Polcher, M. Braun, M. Debald, N. B. Serce, W. Kuhn, G. Brunagel-Walgenbach and C. Rudlowski, "Detection of Lymphovascular Invasion by D2-40 (Podoplanin) Immunoexpression in Endometrial Cancer," International Journal of Gynecological Cancer, Vol. 22, No. 8, 2012, pp. 1442-1448. http://dx.doi.org/10.1097/IGC.0b013e318269139b

[20] T. Boren, Y. Xiong, A. Hakam, R. Wenham, S. Apte, Z.
Wei, S. Kamath, D. T. Chen, H. Dressman and J. M. Lancaster, "MicroRNAs and Their Target Messenger RNAs Associated with Endometrial Carcinogenesis," Gynecologic Oncology, Vol. 110, No. 2, 2008, pp. 206-215. http://dx.doi.org/10.1016/i.ygyno.2008.03.023

[21] W. Wu, Z. Lin, Z. Zhuang and X. Liang, "Expression Profile of Mammalian microRNAs in Endometrioid Adenocarcinoma," European Journal of Cancer Prevention, Vol. 18, No. 1, 2009, pp. 50-55. http://dx.doi.org/10.1097/CEJ.0b013e328305a07a

[22] T. K. Chung, T. H. Cheung, N. Y. Huen, K. W. Wong, K. W. Lo, S. F. Yim, N. S. Siu, Y. M. Wong, P. T. Tsang, M. W. Pang, M. Y. Yu, K. F. To, S. C. Mok, V. W. Wang, C. Li, A. Y. Cheung, G. Doran, M. J. Birrer, D. I. Smith and Y. F. Wong, "Dysregulated Micrornas and Their Predicted Targets Associated with Endometrioid Endometrial Adenocarcinoma in Hong Kong Women," International Journal of Cancer, Vol. 124, No. 6, 2009, pp. 1358-1365. http://dx.doi.org/10.1002/ijc.24071

[23] E. Bignotti, M. Ragnoli, L. Zanotti, S. Calza, M. Falchetti, S. Lonardi, S. Bergamelli, E. Bandiera, R. A. Tassi, C. Romani, P. Todeschini, F. E. Odicino, F. Facchetti, S. Pecorelli and A. Ravaggi, "Diagnostic and Prognostic Impact of Serum HE4 Detection in Endometrial Carcinoma Patients," British Journal of Cancer, Vol. 104, No. 9, 2011, pp. 1418-1425. http://dx.doi.org/10.1038/bjc.2011.109

[24] E. Kalogera, N. Scholler, C. Powless, A. Weaver, R. Drapkin, J. Li, S. W. Jiang, K. Podratz, N. Urban and S. C. Dowdy, "Correlation of Serum HE4 with Tumor Size and Myometrial Invasion In Endometrial Cancer," Gynecologic Oncology, Vol. 124, No. 2, 2012, pp. 270-275. http://dx.doi.org/10.1016/j.ygyno.2011.10.025

[25] I. Mutz-Dehbalaie, D. Egle, S. Fessler, M. Hubalek, H. Fiegl, C. Marth and A. Widschwendter, "HE4 Is an Independent Prognostic Marker in Endometrial Cancer Patients," Gynecologic Oncology, Vol. 126, No. 2, 2012, pp. 186-191. http://dx.doi.org/10.1016/j.ygyno.2012.04.022

[26] R. G. Moore, C. M. Miller, A. K. Brown, K. Robison, M. Steinhoff and G. Lambert-Messerlian, "Utility of Tumor Marker HE4 to Predict Depth of Myometrial Invasion in Endometrioid Adenocarcinoma of the Uterus," International Journal of Gynecological Cancer, Vol. 21, No. 7, 2011, pp. 1185-1190. http://dx.doi.org/10.1097/IGC.0b013e3182229ad8

[27] R. Angioli, F. Plotti, S. Capriglione, R. Montera, P. Damiani, R. Ricciardi, A. Aloisi, D. Luvero, E. V. Cafa, N. Dugo, M. Angelucci and P. Benedetti-Panici, "The Role of Novel Biomarker HE4 in Endometrial Cancer: A Case Control Prospective Study," Tumour Biology, Vol. 34, No. 1, 2013, pp. 571-576. http://dx.doi.org/10.1007/s13277-012-0583-0 